\title{
An interferometric ex vivo study of corneal biomechanics under physiologically representative loading, highlighting the role of the limbus in pressure compensation
}

\author{
Abby Wilson ${ }^{1 *}$ D, John Jones ${ }^{2}$, John R. Tyrer ${ }^{1,2}$ and John Marshall ${ }^{3}$
}

\begin{abstract}
Background: The mechanical properties of the cornea are complex and regionally variable. This paper uses an original method to investigate the biomechanics of the cornea in response to hydrostatic loading over the typical physiological range of intra-ocular pressure (IOP) fluctuations thereby increasing understanding of clinically relevant corneal biomechanical properties and their contributions to the refractive properties of the cornea.

Methods: Displacement speckle pattern interferometry (DSPI) was used to measure the total surface displacement of 40 porcine and 6 human corneal-scleral specimens in response to pressure variations up to $1 \mathrm{mmHg}$ from a baseline of $16.5 \mathrm{mmHg}$. All specimens were mounted in a modified artificial anterior chamber (AAC) and loaded hydrostatically. Areas of high strain in response to loading were identified by comparing the displacements across different regions.

Results: The nature of the response of the corneal surface to loading demonstrated high regional topographic variation. Mechanical properties were shown to be asymmetrical, and deformation of the limbal and pre-limbal regions dominated these responses respectively with over $90 \%(\mathrm{~N}-\mathrm{T})$ and $60 \%(\mathrm{~S}-\mathrm{I})$ of the total maximum displacement occurring in these regions indicating high-strain. In contrast, the curvature of the central cornea remained relatively unchanged merely translating in position.

Conclusions: The limbal and pre-limbal regions of the cornea appear to be fundamental to the absorption of small pressure fluctuations facilitating the curvature of the central cornea to remain relatively unchanged. The differential mechanical properties of this region could have important implications for the application of corneal surgery and corneal crosslinking, warranting further investigation.
\end{abstract}

Keywords: Cornea, Biomechanics, Interferometry, Limbus, Topography

\footnotetext{
* Correspondence: a.wilson9@lboro.ac.uk

'Wolfson School of Mechanical, Manufacturing and Electrical Engineering, Loughborough University, Loughborough, UK

Full list of author information is available at the end of the article
}

C C The Author(s). 2020 Open Access This article is licensed under a Creative Commons Attribution 4.0 International License, which permits use, sharing, adaptation, distribution and reproduction in any medium or format, as long as you give appropriate credit to the original author(s) and the source, provide a link to the Creative Commons licence, and indicate if changes were made. The images or other third party material in this article are included in the article's Creative Commons licence, unless indicated otherwise in a credit line to the material. If material is not included in the article's Creative Commons licence and your intended use is not permitted by statutory regulation or exceeds the permitted use, you will need to obtain permission directly from the copyright holder. To view a copy of this licence, visit http://creativecommons.org/licenses/by/4.0/ The Creative Commons Public Domain Dedication waiver (http://creativecommons.org/publicdomain/zero/1.0/) applies to the data made available in this article, unless otherwise stated in a credit line to the data. 


\section{Introduction}

The biomechanics of the cornea govern its shape and hence its refractive power. Understanding how the mechanical properties of the cornea contribute to its shape has become of increasing importance since the advent and rapid dissemination of refractive surgery in the 1990's, as the introduction of surgical incisions to the cornea results in modifications to its mechanical properties $[1,2]$. It is now even more pertinent given the possibility of direct topographic manipulation of corneal stiffness via corneal crosslinking (CXL) [3], which has demonstrated potential as a minimally-invasive means to provide small refractive correction [4]. However, there have been major limitations associated with the techniques employed for biomechanical assessment in basic and clinical studies, and to date, there is no established method for evaluating the mechanics of the cornea and generating spatially resolved information that is relevant to the clinician.

The cornea is a structurally complex, viscoelastic membrane with regionally variable, strain dependent biomechanical properties; hence, many factors such as the strain rate, hydration and direction of the applied force, can lead to variations in response [5]. To gain a useful understanding of how biomechanics govern corneal shape and behaviour and predict how the biomechanical properties of the tissue may change following disease, trauma or surgical intervention, it is necessary to consider the mechanics of the entire cornea in response to physiologically-relevant forces.

However, up to the present time, the majority of studies on corneal biomechanics have been performed ex vivo, using strip extensometry to evaluate corneal elasticity expressed in terms of the elastic moduli (Young's, tangent, secant) of excised strips of corneal tissue [6-8]. Whilst this technique has advantages in terms of its simplicity, low cost, and ability to provide a quantitative measure of stiffness, it also has many limitations. Principally, strips are isolated from the dome of the cornea, compromising the integrity of its microstructure, and removing the natural physiological state of tension and shape that plays an important role in governing the biomechanics of the tissue in vivo. This, in addition to the difficulties associated with maintaining a strip of tissue at physiological hydration during the measuring procedure; and the fact strains generally far exceed those experienced physiologically contributes to the substantial variation in elastic moduli that have been reported in the literature, with values ranging from $1.3 \mathrm{MPa}$ [9] to $57 \mathrm{MPa}$ [10]. Hence, despite extensometry testing providing a useful and simplistic measure of changes to tissue elasticity in the case of interventions such as CXL, it would be erroneous to assume that stiffness and tensile properties measured via this method are truly applicable to whole-tissue models of corneal biomechanics.
Acknowledging the limitations of strip testing, a variety of methods have now been investigated to probe the biomechanics of intact corneal tissue under physiologically relevant loading conditions, these methods include; Brillouin spectroscopy (BrS), optical coherence elastography (OCE), high frequency ultrasound (HFU), digital image correlation (DIC) and interferometry. Of these, $\mathrm{BrS}$ and OCE have demonstrated potential to acquire data in a clinical setting [11, 12]. BrS evaluates longitudinal modulus, which is related to compressibility (bulk modulus) in isotropic materials. However, in hydrated materials such as the cornea, $\mathrm{BrS}$ signals represent a volume-weighted aggregate longitudinal modulus of the fluid and solid components of a tissue, often dominated by the fluid component (which for the cornea is spatially and temporally variable). As a result, Brillouin spectra do not correlate directly with elastic properties, recently leading to one group cautioning against its use as an optical elastography tool for biological materials [13].

Furthermore, both BrS and OCE are scanning based methods, so there is an inherent trade-off between spatial resolution and acquisition time, with BrS requiring several minutes to generate a relatively lowresolution, limited area, 40-point scan [11]; and OCE requiring around $5 \mathrm{~min}$ to obtain high-resolution 3D data [14]. A more prominent limitation of OCE is a loss of speckle contrast at the corneal periphery limiting data acquisition to the central $\sim 8 \mathrm{~mm}$ [14], unless the cornea is rotated with respect to the image system, leading to longer acquisition times and complexities with image stitching during post-processing. HFU has recently demonstrated potential to measure corneal strain in response to changes in IOP over the cardiac cycle [15], however this method also requires scanning, with data only presented so far for the central region of 2D cross-sections $[15,16]$. If clinical translation is to be realised using either OCE or HFU, further work is required to extend the region of measurement and to account for eye movement over the measurement time.

In contrast to the above methods, DIC and Interferometry are snapshot methods where whole-field data can be captured over a single measurement, requiring only milliseconds. DIC has successfully been used ex vivo to examine regional differences in surface displacement in response to loading $[17,18]$, however displacement sensitivity is limited relative to interferometric techniques. Using speckle interferometric methods, it is possible to generate high spatial resolution maps of corneal and scleral surface deformation, with a displacement sensitivity of 10's of nanometres, over a single measurement taking only milliseconds. Displacement speckle pattern interferometry (DSPI) has previously been used for the examination of ovine corneas [1], where it was used to quantify the effects of refractive surgery on biomechanics. Radial 
speckle pattern shearing interferometry has also been employed to examine age related stiffening [19] and the biomechanical effects of CXL [3], however this particular method is inherently limited by a lack of sensitivity at the central cornea. Despite significant advantages in terms of acquisition time, both DIC and interferometry measure the surface movement, hence the bulk movement of the cornea; they cannot provide detail on any compression that may occur through the thickness of the tissue.

Clinical assessment of corneal biomechanics currently relies on evaluation of the response of the cornea to an air-puff directed at its centre, either via the ocular response analyser (ORA) [20] or the dynamic scheimpflug tonometer (DST) [21]. Although DST has recently demonstrated efficacy for the early detection of biomechanical abnormality [22], neither of these methods directly asses corneal stiffness and cannot realistically predict movement parameters outside the location of the airpuff.

In the present study, DSPI was used to obtain noncontact, non-destructive measurement of full-corneal surface deformation in response to hydrostatic pressure changes designed to simulate small changes in IOP, such as those that occur during the cardiac cycle [23]. Building upon previous work [1], where only a single component of deformation was analysed, here DSPI was combined with subtractive cross-sectional imaging enabling the total set of deformation to be estimated.

\section{Methods and materials}

\section{Measurement and loading system}

The working principles of DSPI are diagrammatically summarised in Supplementary Figure 1. During DSPI, the surface of the target is illuminated with coherent light. In the presence of surface height variations greater than the wavelength of the illumination source, light scattered from the surface of interest has a speckled appearance. The light scattered from this surface is interfered with that of a reference and imaged on a detector to create an interferogram. The speckle pattern formed is unique to that object at the time of measurement, should the surface move, this speckle pattern will change. Capturing a speckle pattern of the object in its reference state and then digitally subtracting subsequent speckle patterns captured as the object moves results in the formation of interference patterns consisting of light and dark fringes, which, with knowledge of the optical set-up, can be mathematically analysed to quantify the surface displacement [24], which when combined with phase-shifting provides sensitivity to displacement on a scale of 10's of nanometres [25].

The layout of the interferometer that was used in this study for the measurement of surface deformation, and the artificial anterior chamber (AAC) onto which corneas were mounted are shown in Fig. 1a and b, respectively. For the interferometry system, illumination was via a diode pumped single-mode solid-state laser $(\lambda=$ $532 \mathrm{~nm}$ ) (06-DPL, Cobolt AB, Solna, SE). The laser was expanded and collimated to a diameter of $25 \mathrm{~mm}$. The illumination beam was passed through a 50:50 beamsplitter with half directed towards the corneal surface and half towards a planar mirror attached to a piezo-electric transducer which was used to generate a phase-stepped reference beam. The beams from the object and the reference were imaged using a CMOS camera with a resolution of 1296 by 972 pixels (CMOS Aptina MT9P031, Basler AG, Ahrensburg, DE) through a $12.5 \mathrm{~mm}-75 \mathrm{~mm}$ zoom lens (C31204, Pentax, Tokyo, JP).

The configuration of the interferometric system, with illumination and imaging directed normal to the surface of the target dictates that the sensitivity is to out-ofplane deformation only (Fig. 1c) and this is constant across the surface of the object. However, due to the curved nature of the cornea, the vector of total displacement is likely to vary across the surface. Therefore, to completely define the deformation of the corneal surface, it was necessary to define the out-of-plane and horizontal and vertical in-plane components of deformation. This was impractical to achieve using interferometry on a curved surface, due to the requirement for offaxis illumination and imaging, hence, problems with shading and uneven illumination. Consequently, estimations for the in-plane component of deformation were made using subtractive imaging of the central crosssection of a cornea deforming under a larger change of pressure $(<20 \mathrm{mmHg})$ and tracking the angle of surface movement via tracking the movement of recognisable regions on the corneal surface, identifiable due to the roughness of the applied surface coating.

Pressure in the AAC was controlled via a reservoir supplying fluid to the closed chamber. During testing, the reservoir and the chamber were filled with Phosphate Buffered Saline (PBS) solution (Sigma-Aldrich, $\mathrm{UK}, \rho=0.995 \mathrm{~g} / \mathrm{ml}$ at $25^{\circ} \mathrm{C}$ ). The reservoir was raised $225 \mathrm{~mm}$ above the surface of the cornea to provide a baseline pressure of $16.5 \mathrm{mmHg}$, within the representative range of normal IOP in both human and porcine eyes [26-28]. To raise and lower the pressure from this baseline, the height of the chamber was adjusted electronically via attachment of the reservoir to a motorised vertical translation stage capable of pressure variations up to a maximum of $4 \mathrm{mmHg}$ above the baseline pressure.

\section{Validation experiments}

To establish that the interferometer was functioning correctly, prior to corneal measurement, two initial experiments were conducted. The first experiment was on a 


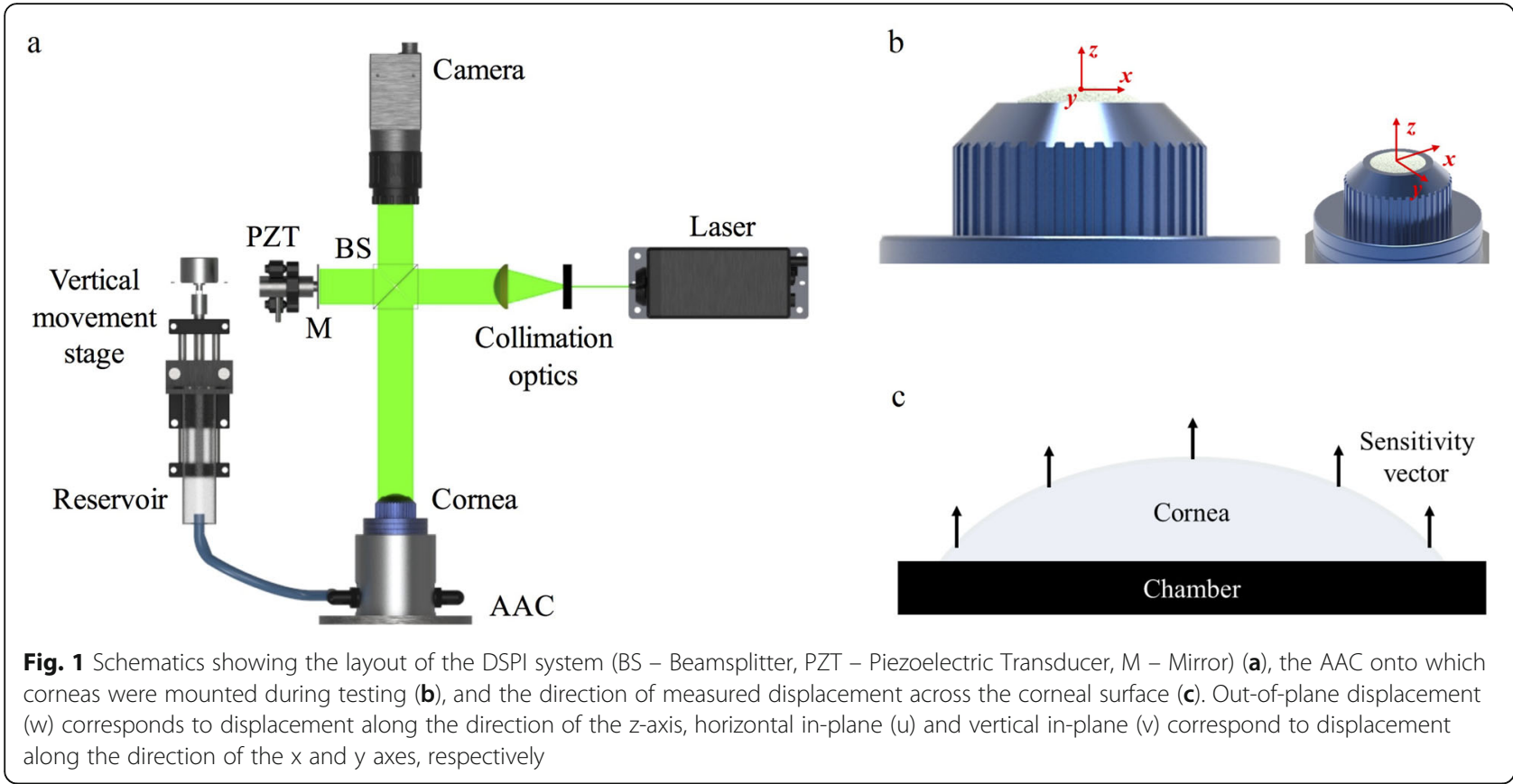

circularly clamped steel plate, centrally point-loaded with a micrometre screw. A pre-load was applied, and a reference image was acquired, the plate was then displaced by $1 \mu \mathrm{m}$ at the centre via movement of the micrometre screw and data was acquired, 10 repeated measurements were taken. Following this, measurements were conducted on a curved silicone hemispherical membrane of uniform thickness (Mini Semisphere mould, Silikomart, IT, $r_{c}=15 \mathrm{~mm}$ ) mounted in the AAC to check that the AAC did not induce any abnormal boundary effects. In this case the membrane was set under an initial pressure of $16.50 \mathrm{mmHg}$ and hydrostatic pressure was increased by $1 \mathrm{mmHg}$ over the course of measurement.

\section{Corneal preparation and testing}

Fresh porcine eyes $(<12 \mathrm{~h}$ post-mortem) with a clear cornea and intact epithelium were obtained from a local abattoir (Joseph Morris Butchers, South Kilworth, UK). The eyes were stored in a moist, sealed container and refrigerated at $4{ }^{\circ} \mathrm{C}$ until measurement. All porcine eyes were used within 3 days of slaughter. Prior to measurement, the corneas were isolated from the posterior globe, leaving a $3 \mathrm{~mm}$ scleral border for mounting. For the human eye experiments, 6 human corneal-scleral specimens from 3 donors were obtained from Moorfield's Biobank (UCL Institute of Ophthalmology, London, UK), with ethical approval granted by Moorfields Biobank internal ethics committee (Ref: 10/ H0106/57-2015ETR45). The human corneas were surplus for donor purposes and had been collected with permission for research use. The corneal specimens had been in storage for over 8 weeks prior to being released for research use. The human corneas were suspended in organ donor culture $(80 \mathrm{ml}$ Eagle's minimum essential medium with HEPES buffer, 26 $\mathrm{mmol} / \mathrm{l} \mathrm{NaHCO} 3,2 \%$ foetal bovine serum, $2 \mathrm{mmol} / \mathrm{l}$ L-glutamine, penicillin, streptomycin and amphotericin B). All corneas remained in the solution until required for measurement.

All corneas were de-epithelialized prior to measurement. The epithelium has previously been shown to have a negligible contribution to stiffness [29], and its removal prevented any changes to the epithelium, that may have occurred during storage, from affecting the measured response. The corneal-scleral specimens were then mounted within the AAC, which imposed a fixed boundary on the scleral region $1.5 \mathrm{~mm}$ to $3.0 \mathrm{~mm}$ outside the edge of the cornea. As the cornea is designed to transmit optical radiation, surface reflection was small and had to be enhanced by means of a scattering agent. The corneas were coated with a thin layer of hollow glass microspheres - Sphericel 110P8 (Potters Ind. LLC, PA, USA), to amplify the scatter from the surface. Prior to loading and measurement, the corneas were set under the baseline pressure of $16.5 \mathrm{mmHg}$ and maintained under this constant pressure for 30-min to allow for stress-relaxation. During testing, each cornea underwent 3 loading cycles during which the pressure was raised by $0.25,0.5,0.75$ and $1 \mathrm{mmHg}$ in turn. At the end of each loading increment, the movement of the reservoir was paused for $0.5 \mathrm{~s}$ during which data was captured and processed, and after which the pressure was restored to baseline. 


\section{Data analysis}

The out-of-plane displacement $(w)$ was calculated from the measured phase change due to deformation $\left(\Delta \phi_{d e f}\right.$ (radians)) using eq. 1 [24], where the wavelength of the illumination source $(\lambda)$ was $532 \mathrm{~nm}$.

$$
w=\Delta \phi_{\text {def }} \cdot \frac{\lambda}{4 \pi}
$$

The horizontal in-plane (u) and vertical in-plane (v) displacement components were estimated using the measured out-of-plane displacement component $(\mathrm{w})$ and the angle of deformation estimated from subtractive cross-sectional imaging as described by eqs. 2 and 3, where $\theta_{\mathrm{x}}$ and $\theta_{\mathrm{y}}$ are the angles of deformation with respect to the horizontal and vertical axes, respectively.

$$
\begin{aligned}
& u=w \tan \theta_{x} \\
& v=w \tan \theta_{y}
\end{aligned}
$$

\section{Results}

\section{Loading plate and response of uniform membrane}

The average central displacement of the clamped steel plate loaded via the micrometre screw was as expected at $0.98 \mu \mathrm{m}(\mathrm{SD} \pm 0.02 \mu \mathrm{m})$. The deformation of the curved homogenous membrane (Fig. 2a) also showed the expected distribution, with zero deformation at the fixed boundary, maximum displacement at the central point furthest from the fixed boundary (Fig. 2b) and a relatively constant gradient to the rate-of-change of displacement (Fig. 2c).

\section{Regional differences in corneal response}

In contrast to the homogenous membrane (Figs. 2 and 3a), corneas were found to demonstrate high regional variability in response to pressure changes (Figs. 3b and 4). In both the human and porcine corneas examined, there was a tendency for deformation to be concentrated in the limbal region. This was evident due to the high fringe concentration observed in this region, relative to other regions during loading (Fig. 3b) which was in clear contrast to the response of the homogenous membrane, which when mounted in the AAC, showed relatively even fringe spacing (Fig. 3a). Furthermore, a common trend was observed with $23 / 40$ (57\%) porcine corneas and 4/6 (67\%) of human corneas showing similarities in the pattern of regional displacement.

A typical response of a human cornea is shown in Fig. 4 (responses of all human corneas are provided in Supplementary Figures 2 and 4). In these specimens, the response was asymmetrical; the rate of change of displacement across the central 8 to $10 \mathrm{~mm}$ of the cornea was close to zero (Table 1), especially across the nasaltemporal axis (Fig. 4d) indicating minimal changes in curvature across this region; and the highest rate of change of displacement was at the edge of the cornea in the limbal region (Fig. $4 \mathrm{~d}$ and $\mathrm{f}$ ), where the cornea joins the sclera. Taken together, these indicate a high strain concentration at the limbal/prelimbal region, confirmed by the relatively higher values for $\frac{\delta w}{\delta x}$ and $\frac{\delta w}{\delta y}$ here when compared to the central $8 \mathrm{~mm}$ of the N-T and S-I axes (Table 1). It was also observed that while the magnitude of $\frac{\delta w}{\delta x}$ and $\frac{\delta w}{\delta y}$ increased significantly in the limbal region in response to larger pressure variations (0.5 to 1.0

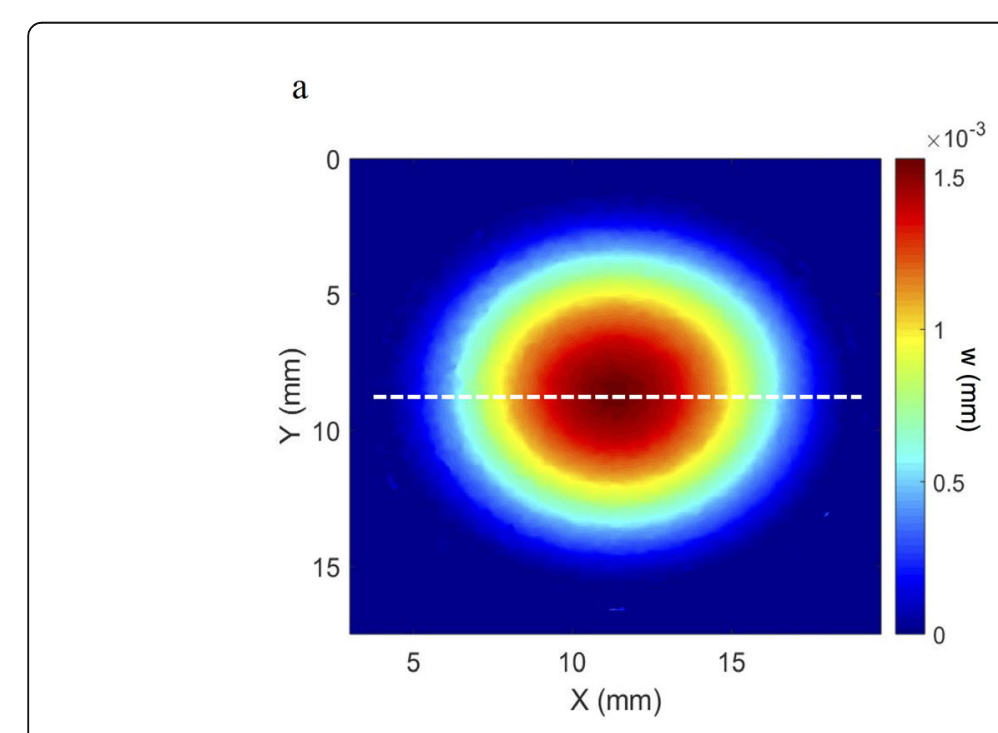

$\mathrm{b}$

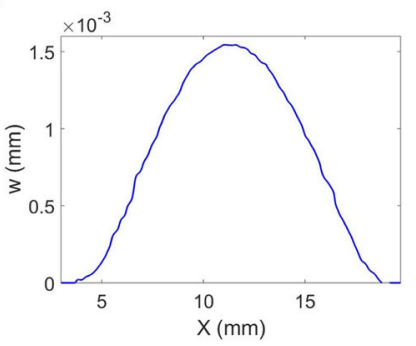

$\mathrm{C}$

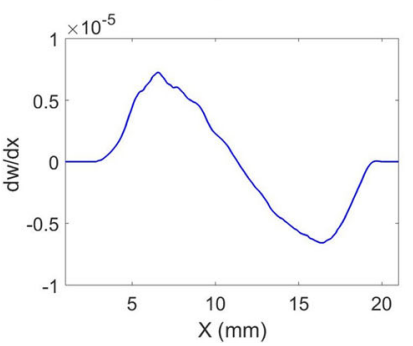

Fig. 2 Out-of-plane surface displacement of a curved silicone hemispherical membrane in response to hydrostatic pressure increase from 16.5 $\mathrm{mmHg}$ to $17.5 \mathrm{mmHg}$; whole surface displacement map (a); cross-section of out-of-plane displacement along dashed line (b); cross-section of gradient of out-of-plane displacement along dashed line (c) 

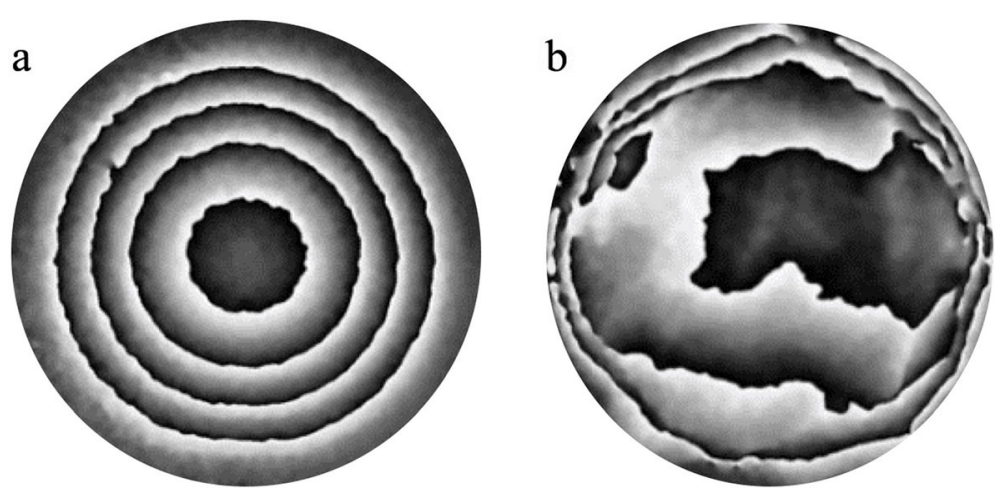

Fig. 3 Fringe distributions obtained from a curved silicone hemispherical membrane (a) and a human cornea (b) in response to a pressure increase from $16.5 \mathrm{mmHg}$ to $17.0 \mathrm{mmHg}$

$\mathrm{mmHg}$ ), across the centre $\frac{\delta w}{\delta x}$ and $\frac{\delta w}{\delta y}$ remained almost constant, showing minimal changes to the curvature of this region across the full pressure range. In general, over $90 \%$ (N-T axis) and $60 \%$ (S-I) of the maximum outof-plane displacement occurred in the outer $2 \mathrm{~mm}$ of the human corneas incorporating the limbal region (Table 1). A typical response for a porcine cornea is shown in Supplementary Figure 3. As with human corneas, the majority of strain was concentrated at the limbus however, in porcine corneas, deformation was greater in the peripheral cornea at the superior and inferior poles when compared to the nasal temporal.
The corneas that did not show this typical distribution of displacement can be grouped into two main categories. A few porcine corneas (5/40) showed a similar displacement distribution to the one previously described, but rotated through $90^{\circ}$, suggesting that this could have occurred due to errors in determining initial corneal orientation. The majority of the other corneas tested (12/40 porcine corneas and 2/6 human corneas), showed a distribution where the maximum rate of change of displacement remained at the limbal region, but instead of maintaining an overall relatively constant level of displacement across the central region, the displacement
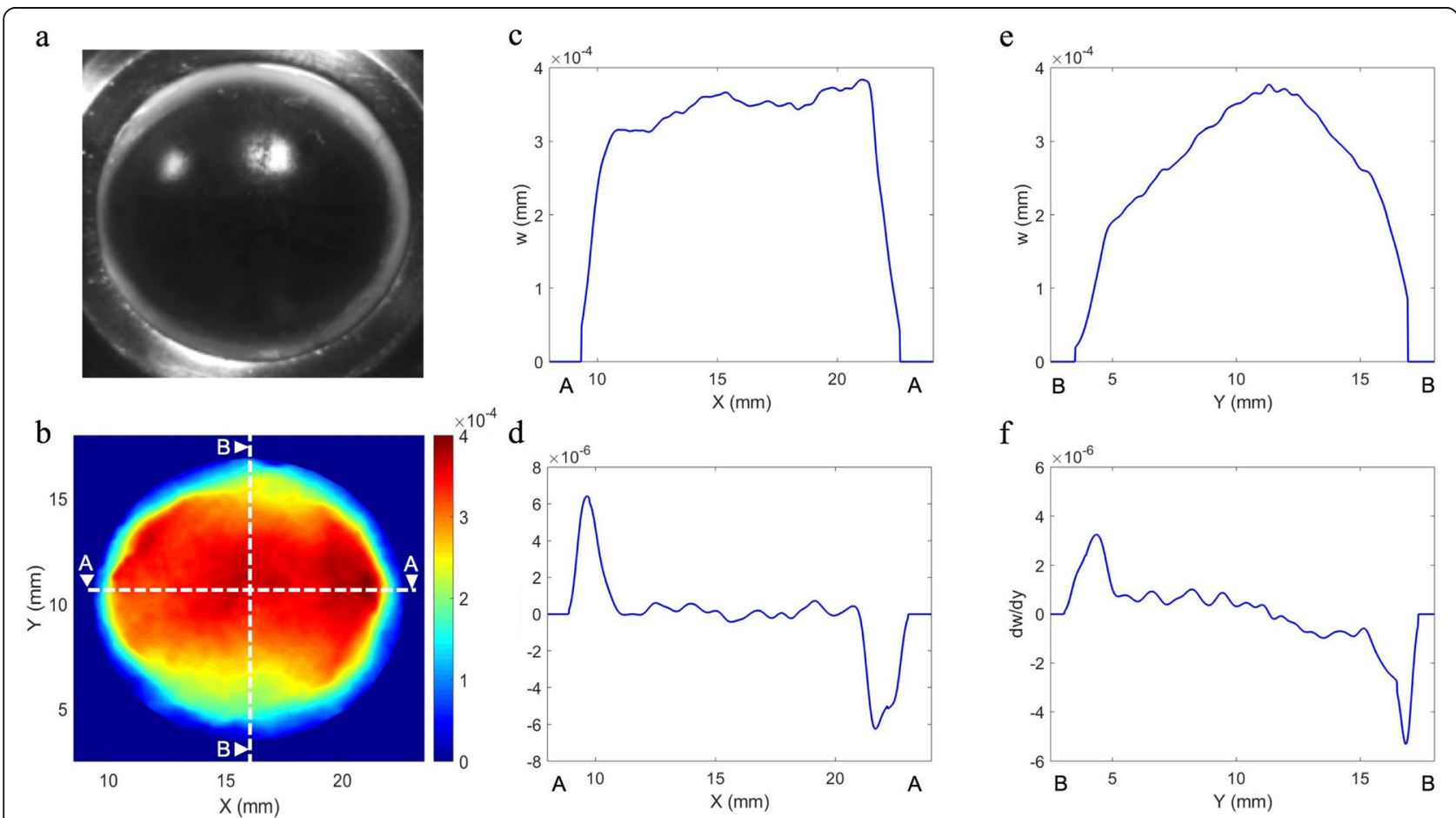

Fig. 4 Typical response of a human cornea to a pressure increase from $16.5 \mathrm{mmHg}$ to $16.75 \mathrm{mmHg}$. Photograph of cornea in AAC for positional reference (a), full-surface map of out-of-plane displacement (b); out-of-plane displacement across section A-A (c); gradient of out-of-plane displacement across section A-A (d); out-of-plane displacement across section B-B (e); gradient of out-of-plane displacement across section B-B (f) 
Table 1 Comparison of out-of-plane displacement of human corneas at the central cornea and at the limbus in response to a 0.25 $\mathrm{mmHg}$ increase in hydrostatic pressure

\begin{tabular}{|c|c|c|c|c|c|c|c|}
\hline $\begin{array}{l}\text { Human } \\
\text { cornea } \\
\text { No. }\end{array}$ & $\begin{array}{l}\text { W at central } \\
\text { cornea, mean } \pm \\
\text { SD }(\mu \mathrm{m})\end{array}$ & $\begin{array}{l}\text { w at limbal/ } \\
\text { prelimbal region } \\
(\mathrm{N}-\mathrm{T} \text { axis) }(\mu \mathrm{m})\end{array}$ & $\begin{array}{l}\text { w at limbal/ } \\
\text { prelimbal region } \\
(\mathrm{S}-\mathrm{I} \text { axis })(\mu \mathrm{m})\end{array}$ & $\begin{array}{l}\text { Mean } \mathrm{dw} / \mathrm{dx} \\
\text { (central } 8 \mathrm{~mm} \mathrm{~N}-\mathrm{T} \\
\text { axis) } \times 1.0 \mathrm{e}^{-06}\end{array}$ & $\begin{array}{l}\text { Mean dw/dx } \\
\text { (limbus N-T axis) } \\
\times 1.0 \mathrm{e}^{-06}\end{array}$ & $\begin{array}{l}\text { Mean } \mathrm{dw} / \mathrm{dy} \\
\text { (central } 8 \mathrm{~mm} \text { S-I } \\
\text { axis) } \times 1.0 \mathrm{e}^{-06}\end{array}$ & $\begin{array}{l}\text { Mean dw/dy } \\
\text { (limbus S-I axis) } \\
\times 1.0 \mathrm{e}^{-06}\end{array}$ \\
\hline 1 & $0.46 \pm 0.058$ & 0.46 & 0.44 & 0.66 & 3.39 & 0.74 & 3.59 \\
\hline 2 & $0.29 \pm 0.036$ & 0.27 & 0.20 & 0.31 & 4.21 & 0.57 & 2.88 \\
\hline 3 & $0.35 \pm 0.028$ & 0.37 & 0.27 & 0.26 & 4.39 & 0.57 & 1.91 \\
\hline 4 & $0.68 \pm 0.050$ & 0.46 & 0.42 & 1.22 & 3.87 & 1.16 & 3.91 \\
\hline 5 & $0.71 \pm 0.068$ & 0.66 & 0.63 & 0.58 & 5.70 & 0.81 & 5.13 \\
\hline
\end{tabular}

tended to be greater in one particular region, indicating localised regions of biomechanical weakness. The position of this region varied between corneas. In one human cornea, this area of weakness was clearly identifiable as damage introduced from the insertion of the surgical thread used to suspend the cornea in the transplant solution (Supplementary Figure 4). Discounting this cornea, the magnitude of out-of-plane displacement at the central cornea for the remaining human corneas tested in response to a $0.25 \mathrm{mmHg}$ change in hydrostatic pressure are shown in Table 1 and ranged from $0.29 \mu \mathrm{m}$ to $0.71 \mu \mathrm{m}$ (median $0.50 \mu \mathrm{m}$ ). Assuming linearity in response, this corresponds to apical displacement of $3.6 \mu \mathrm{m}$ to $10.4 \mu \mathrm{m}$ over a normal range of ocular pulse amplitudes $(1.8-4.3 \mathrm{mmHg})$ [23] consistent with a recent study examining the deformation of a whole eye-globe over a physiological range of pressure variations [18].

\section{Directional aspects of response}

To examine the directional aspects of the response, the deformation of the central-cross-section of the corneas in response to a larger pressure change $(<20 \mathrm{mmHg})$

a

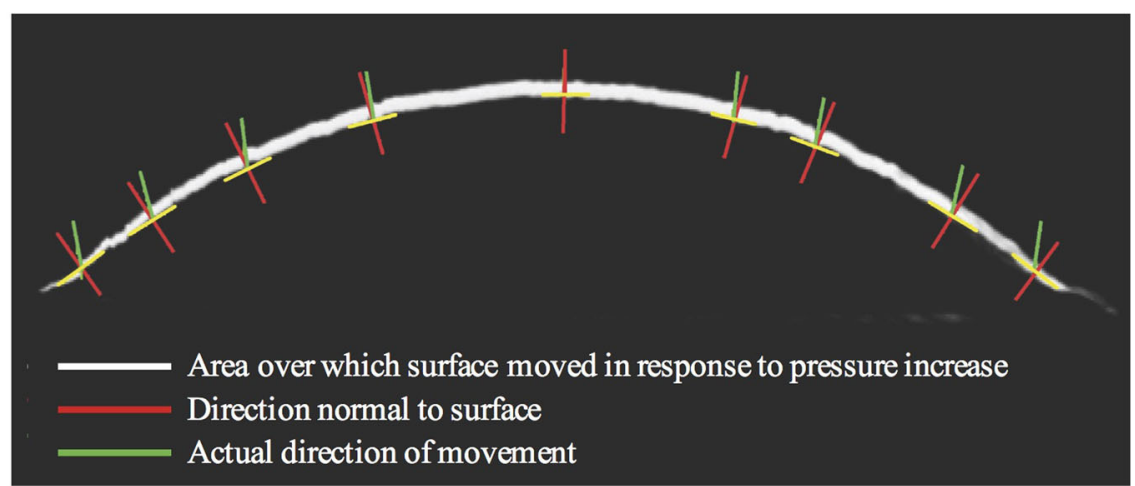

b

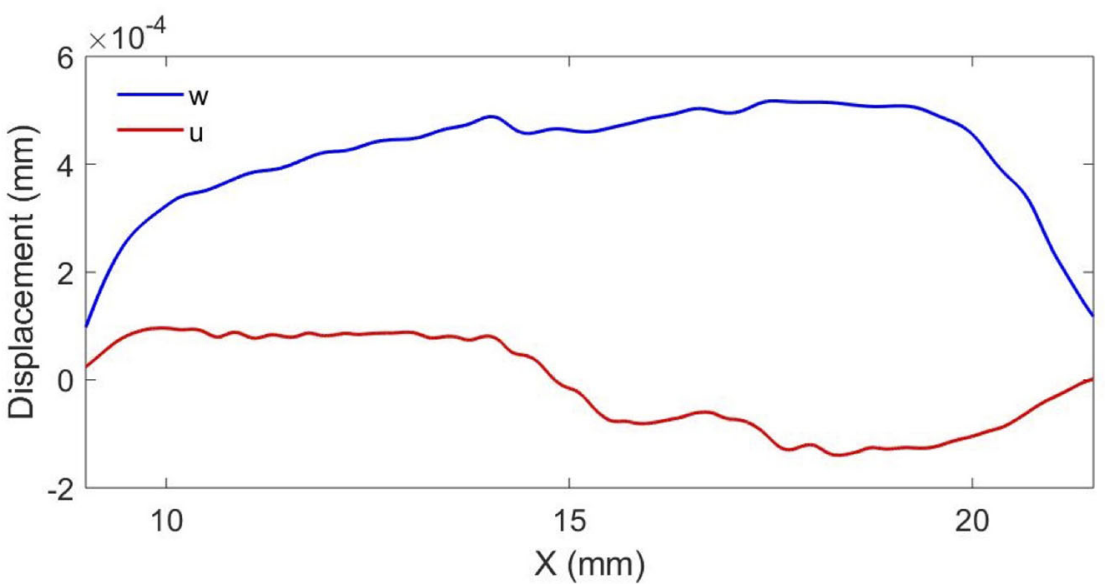

Fig. 5 Subtractive video recording of the central cross-section of a cornea deforming in response to a pressure increase (a), comparison of measured out-of-plane and estimated horizontal in-plane displacement along central N-T cross section of a human cornea (b) 
was recorded (Fig. 5). From this, it was identified that displacement tended to occur predominantly out-ofplane in comparison to that which would be predicted for an isotropic hemisphere where it would be expected to occur in a direction normal to the surface. The estimated in-plane component calculated using the angle of deformation and the measured out-of-plane component is shown in Fig. 5b. The in-plane contribution to overall displacement was relatively small, especially over the central $3 \mathrm{~mm}$ of the cornea and was approximately uniform either side of this over the central $\sim 8 \mathrm{~mm}$ of the cornea. Hence, $\frac{\delta u}{\delta x} \approx 0$ over this region, and thus the measured differences in the out-of-plane displacement component may be considered predominately representative of changes in central curvature.

\section{Discussion}

The distribution of the observed displacement indicates that the biomechanics of the limbal region are fundamental to the response of the cornea to small pressure perturbations. Based on the data presented here, it is postulated that the limbus behaves akin to a dashpot, straining in the direction perpendicular to the circumferential orientation of collagen fibres in this region to absorb pressure increases (Fig. 6), facilitating the central optical zone to maintain a relatively constant curvature. Professor Keith Meek has previously put forward an identical hypothesis of limbal deformation (unpublished) to that shown in Fig. 6 as a result of his structural studies, but the present paper has been the first to provide empirical data on human cornea to support this mode of deformation. In addition, several previous studies have demonstrated similar evidence of a region of increased compliance approaching the limbal junction $[17,18,30-$ 32], whilst others have confirmed minimal changes occur to central corneal curvature in response to IOP changes [33, 34].

Structural aspects, including collagen distribution and orientation; and the distribution and concentration of elastin fibres, appear to support this mode of deformation. Both human and porcine corneas have an annulus of circumferentially aligned collagen at the limbus [35], an arrangement which would be expected to provide high circumferential strength to support the propensity towards out-of-plane motion (Fig. 5a) observed at the edge of the cornea. The increased thickness and collagen density in this region would also indicate that it is adept for dealing with stress, as the increased thickness of the tissue acts to minimise strain concentrations. However, strains were not quantified directly in this study due to an absence of through thickness information. An initial concern was that the fixed boundary constraints imposed on the sclera could have affected the motion of the cornea in regions within close proximity to the chamber. However, the experiments with a homogenous membrane did not indicate an obvious effect (Fig. 2) and results from a study by Smolek [31] on human eye globes using holography, showed similar deformation patterns for corneas that remained as a part of the eye globe.

Collagen crimp is a further feature of the cornea that has recently been postulated to play a key role in its mechanical behaviour [36], with a degree of fibrillar elongation via straightening of collagen crimp suggested to act complementary to the action of elastic fibres in the accommodation of small pressure perturbations [36]. Crimp parameters have been shown to vary significantly across the cornea [37, 38], suggesting a non-uniform response to loading, but studies have yet to examine regional modifications to collagen crimp under loading.

With respect to elastin fibres, studies have shown that they are most concentrated and thickest in the posterior limbus and peripheral cornea [39-41]. Here, they are present as elastin sheets, most concentrated in a layer above Descemet's membrane, likely originating from the posterior limbus and adjacent sclera. From these sheets narrower fibres then extend towards the central cornea. The majority of fibres are reported to run radially or obliquely towards the limbus whilst others have been reported to run parallel to the circumferentially aligned collagen at the limbus [40]. Both the presence of elastin fibres and the elastin content has been confirmed to decrease towards the central regions of the cornea [41], again indicating that both the limbus and the peripheral cornea likely play a key role in the compensation of small pressure fluctuations.
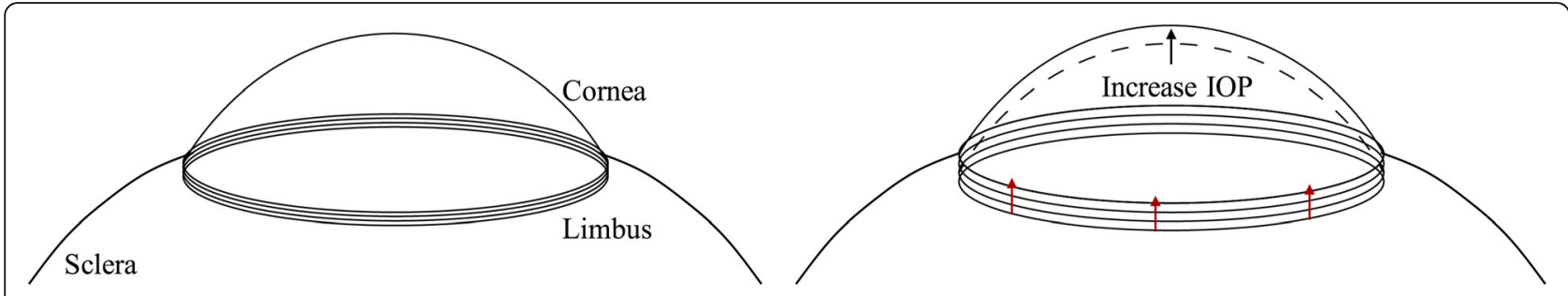

Fig. 6 Hypothesised predominant mode of deformation in response to small pressure perturbations 
Two additional aspects of the response that were highlighted from the displacement maps obtained on human and porcine corneas were differences in the behaviour of the superior-inferior (S-I) axis and nasaltemporal (N-T) axis, and a lack of symmetry in the response with respect to each axis. For human corneas, the magnitude of displacement across the $\mathrm{N}-\mathrm{T}$ axis changed very little inside the limbus, whereas for the S-I axis, the magnitude of displacement very gradually increased towards the centre, suggesting that this axis was slightly less resistant to changes in curvature. Structural studies have identified differences between these two axes. Visually, it can be seen that there is an extension of limbal tissue onto the cornea at the top and bottom of the S-I axis, making it appear shorter than the N-T [42]. Differences have also been identified in the transitioning of collagen at the limbus in human corneas [43]. The $\mathrm{N}-\mathrm{T}$ axis, having a $1.5 \mathrm{~mm}$ transition zone where the collagen running parallel to this axis, bifurcates to gradually become circumferentially aligned, whereas in the S-I axis, the collagen runs parallel to this axis right up until the limbus. Porcine corneas, have been reported in one study to lack the orthogonal arrangement of collagen across the central cornea [35], favouring a circumferential arrangement across the tissue. This could partially account for some of the differences seen in the response between human and porcine corneas. Based on the previous discussion, it would be of interest to examine differences, if any, in the elastic fibre network at the limbal junction with respect to these axes.

In terms of axial symmetry, it was evident, in most of the human corneas, that greater deformation tended to occur towards one side of the N-T axis, the specific side remains ambiguous as the position of the specific poles with respect to each axis could not be confirmed. Similarly, previous structural studies have shown that left and right eyes are structurally distinct [44], indicating that the biomechanics are unlikely to be axially symmetrical.

The specific features of the corneal response to small pressure changes that have been described here are highly important and require further investigation across a larger number of human samples. The information that has been demonstrated from this study and could be obtained from future studies using these imaging techniques, has the potential to enhance the accuracy and validity of computational models of corneal biomechanics aimed at predicting the outcomes of surgical procedures and clinical therapies such as CXL.

The non-destructive nature and high sensitivity of the measurement technique used in this study makes it a highly effective method for ex vivo examination of the regional changes to corneal biomechanics that occur due to invasive procedures such as refractive surgery, and due to treatments aimed at introducing biomechanical changes such as CXL. The latter could benefit greatly from spatial quantification of changes to biomechanics as this could enable the development of optimised treatment algorithms to deliver accurate and predictable refractive modifications.

A limitation of the method used is that it only measures surface displacements and therefore does not provide a full assessment of biomechanics. Other techniques have demonstrated compressive strains through the thickness of the cornea in response to physiological loading [15]. Hence, to gain a complete understanding of corneal biomechanics, it may be most effective to use this technique in combination with others, such as OCE or HFU, capable of measuring throughthickness displacements, while maximising the advantages of fast whole-field image acquisition times provided by interferometry.

A further limitation of this study, common with the majority of ex vivo studies, is the challenge in replicating and maintaining physiological levels of hydration in the tissue. Generally, human corneal samples cannot be obtained fresh and storage results in corneal swelling. Since hydration contributes to tissue biomechanics, swelling could lead to changes to the response of the tissue. However, a study had demonstrated only slight differences in deformation patterns with IOP despite significant differences in hydration state [45], and it would be expected that even if changes in the magnitude of deformation changed, the overall mechanism of deformation, with high strain concentrations at the limbus, would remain the same.

Although this study has been ex vivo in nature, it ultimately is necessary for information of this kind, regarding spatial variation in biomechanics, to be measured in vivo. This would facilitate the early detection and optimised treatment of diseases, such as keratoconus, prior to significant changes in topography; and enable individuals that may be at risk of post-LASIK ectasia to be identified, preventing them from undertaking potentially damaging elective treatments. Further, if an individual's corneal biomechanics could be topographically mapped, it would offer the opportunity for delivery of optimised and customised procedures such as laser refractive surgery for astigmatism, and CXL together with adjustments post-cataract astigmatism. Significant efforts are going into the development of a clinical device based on similar principles.

\section{Conclusions}

The stiffness of the cornea is regionally variable. The limbal and pre-limbal regions of the cornea appear to play a key role in the absorption of small pressure fluctuations, facilitating the central cornea to maintain a 
relatively constant curvature. These findings could have important implications for corneal surgery and for the delivery of targetted-CXL, hence further investigations are required on a larger number of human samples.

DSPI has been demonstrated here as an effective technique to examine corneal surface deformation in response to physiological scale pressure variations, and can be used alongside other methods to map corneal biomechanics.

\section{Supplementary information}

Supplementary information accompanies this paper at https://doi.org/10. 1186/s40662-020-00207-1.

Additional file 1: Supplementary Figure 1. Diagrammatic summary of the working principles of displacement speckle pattern interferometry (DSPI)

Additional file 2: Supplementary Figure 2. Surface plots showing out-of-plane displacement of human corneas in response to a pressure increase from $16.50 \mathrm{mmHg}$ to $16.75 \mathrm{mmHg}$. Positions marked with $\mathbf{X}$ relate to locations at which limbal and central displacements were compared in Table 1.

Additional file 3: Supplementary Figure 3. Typical response of a porcine cornea to a pressure increase from $16.50 \mathrm{mmHg}$ to $17.00 \mathrm{mmHg}$. Full surface map of out-of-plane displacement (a), out-of-plane displacement along section A-A (b), out-of-plane displacement along section B-B (c)

Additional file 4: Supplementary Figure 4. Measurement region damaged due to insertion of surgical thread; photograph of cornea whilst suspended in transplant solution (a); map of out-of-plane surface displacement in response to pressure increase from $16.5 \mathrm{mmHg}$ to 17.0 $\mathrm{mmHg}(\mathrm{b})$, area of damage clearly evident as region of increased displacement (dark red region).

\section{Acknowledgments}

Professor Keith Meek for discussions regarding the relationship of corneal structure to biomechanics.

Dr. Sally Hayes for assistance with manuscript preparation.

\section{Authors' contributions \\ Abby Wilson designed the study, was responsible for system design and operation; data acquisition and analysis; interpretation of data; and preparation of the manuscript. John Jones contributed to system design, the creation of software to run the interferometry system and process the data and revision of the manuscript. John $\mathrm{R}$ Tyrer contributed lab facilities and equipment and contributed to the design of the study. John Marshall contributed to the supply of materials, lab facilities, interpretation of the data, design of the study and preparation of the manuscript. The author(s) read and approved the final manuscript.}

\section{Funding}

During the course of this study, Dr. Abby Wilson was enrolled on a PhD, and was funded by EPSRC and Fight for Sight.

\section{Availability of data and materials}

All relevant data generated or analysed during this study are included in this submitted article and its supplementary files. Full imaging datasets are available from the corresponding author on reasonable request.

\section{Ethics approval and consent to participate}

All human tissue used in this study was obtained from Moorfields Biobank with ethical approval granted by Moorfields Biobank internal ethics committee (Ref: 10/H0106/57-2015ETR45).

\section{Consent for publication}

Not applicable.

\section{Competing interests}

The authors declare they have no competing interests.

\section{Author details}

${ }^{1}$ Wolfson School of Mechanical, Manufacturing and Electrical Engineering, Loughborough University, Loughborough, UK. ${ }^{2}$ Laser Optical Engineering Ltd., Derbyshire, UK. ${ }^{3}$ Institute of Ophthalmology, UCL, London, UK.

Received: 13 February 2020 Accepted: 25 July 2020

Published online: 13 August 2020

\section{References}

1. Jaycock PD, Lobo L, Ibrahim J, Tyrer J, Marshall J. Interferometric technique to measure biomechanical changes in the cornea induced by refractive surgery. J Cataract Refract Surg. 2005:31(1):175-84.

2. Wilson A, Marshall J, Tyrer JR. The role of light in measuring ocular biomechanics. Eye (Lond). 2016;30(2):234-40.

3. Knox Cartwright N, Tyrer JR, Marshall J. In vitro quantification of the stiffening effect of corneal cross-linking in the human cornea using radial shearing speckle pattern interferometry. J Refract Surg. 2012;28(7):503-8.

4. Seiler TG, Fischinger I, Koller T, Zapp D, Frueh BE, Seiler T. Customized corneal cross-linking: one-year results. Am J Ophthalmol. 2016;166:14-21.

5. Elsheikh A, Wang D, Pye D. Determination of the modulus of elasticity of the human cornea. J Refract Surg. 2007;23(8):808-18.

6. Hoeltzel D, Altman P, Buzard K, Choe K. Strip extensiometry for comparison of the mechanical response of bovine, rabbit, and human corneas. Trans ASME. 1992;114(2):202-15.

7. Liu X, Wang L, Ji J, Yao W, Wei W, Fan J, et al. A mechanical model of the cornea considering the crimping morphology of collagen fibrils. Invest Opthalmol Vis Sci. 2014;55(4):2739-46.

8. Elsheikh A, Brown M, Alhasso D, Rama P, Campanelli M, Garway-Heath D. Experimental assessment of corneal anisotropy. J Refract Surg. 2008;24(2): $178-87$.

9. Andreassen $\Pi$, Hjorth Simonsen A, Oxlund H. Biomechanical properties of keratoconus and normal corneas. Exp Eye Res. 1980;31(4):435-41.

10. Wollensak G, Spoerl E, Seiler T. Stress-strain measurements of human and porcine corneas after riboflavin-ultraviolet-A-induced cross-linking. J Cataract Refract Surg. 2003;29(9):1780-5

11. Shao P, Eltony AM, Seiler TG, Tavakol B, Pineda R, Koller T, et al. Spatiallyresolved Brillouin spectroscopy reveals biomechanical abnormalities in mild to advanced keratoconus in vivo. Sci Rep. 2019;9(1):7467.

12. De Stefano VS, Ford MR, Seven I, Dupps WJ. Live human assessment of depth-dependent corneal displacements with swept-source optical coherence elastography. PLoS One. 2018;13(12):e0209480.

13. Wu P-J, Kabakova IV, Ruberti JW, Sherwood JM, Dunlop IE, Paterson C, et al. Water content, not stiffness, dominates Brillouin spectroscopy measurements in hydrated materials. Nat Methods. 2018;15(8):561-2.

14. Fu J, Haghighi-Abayneh M, Pierron F, Ruiz PD. Depth-resolved full-field measurement of corneal deformation by optical coherence tomography and digital volume correlation. Exp Mech. 2016;56(7):1203-17.

15. Pavlatos E, Chen H, Clayson K, Pan X, Liu J. Imaging corneal biomechanical responses to ocular pulse using high-frequency ultrasound. IEEE Trans Med Imaging. 2018;37(2):663-70.

16. Clayson K, Pavlatos E, Pan X, Sandwisch T, Ma Y, Liu J. Ocular pulse elastography: imaging corneal biomechanical responses to simulated ocular pulse using ultrasound. Transl Vis Sci Technol. 2020;9(1):5. https://doi.org/10. 1167/tvst.9.1.5.

17. Boyce BL, Grazier JM, Jones RE, Nguyen TD. Full-field deformation of bovine cornea under constrained inflation conditions. Biomaterials. 2008;29(28): 3896-904.

18. Whitford C, Joda A, Jones S, Bao F, Rama P, Elsheikh A. Ex vivo testing of intact eye globes under inflation conditions to determine regional variation of mechanical stiffness. Eye Vis (Lond). 2016;3:21.

19. Knox Cartwright NE, Tyrer JR, Marshall J. Age-related differences in the elasticity of the human cornea. Invest Ophthalmol Vis Sci. 2011;52(7):4324-9.

20. Kotecha A, Russell RA, Sinapis A, Pourjavan S, Sinapis D, Garway-Heath DF. Biomechanical parameters of the cornea measured with the ocular response analyzer in normal eyes. BMC Ophthalmol. 2014;14:11.

21. Ambrósio R Jr, Ramos I, Luz A, Faria FC, Steinmueller A, Krug M, et al. Dynamic ultra high speed Scheimpflug imaging for assessing corneal biomechanical properties. Rev Bras Oftalmol. 2013;72(2):99-102. 
22. Vinciguerra R, Ambrósio R Jr, Roberts CJ, Azzolini C, Vinciguerra P. Biomechanical characterization of subclinical keratoconus without topographic or tomographic abnormalitites. J Refract Surg. 2017;33(6):399-407.

23. Kaufmann C, Bachmann LM, Robert YC, Thiel MA. Ocular pulse amplitude in healthy subjects as measured by dynamic contour tonometry. Arch Ophthalmol. 2006;124(8):1104-8.

24. Petzing JN, Tyrer JR. Recent developments and applications in electronic speckle pattern interferometry. J Strain Anal Eng Des. 1998;33(2):153-69.

25. Xie X, Yang L, Chen X, Xu N, Wang Y. Review and comparison of temporaland spatial-phase shift speckle pattern interferometry for 3D deformation measurement. Sixth Int Symp Precis Mech Meas. 2013;8916:89160D.

26. Murgatroyd H, Bembridge J. Intraocular pressure. Contin Educ Anaesthesia Crit Care Pain. 2008:8(3):100-3.

27. Ruiz-Ederra J, García M, Hernández M, Urcola H, Hernández-Barbáchano E, Araiz J, et al. The pig eye as a novel model of glaucoma. Exp Eye Res. 2005; 81(5):561-9.

28. Schneider $\mathrm{E}$, Grehn F. Intraocular pressure measurement-comparison of dynamic contour tonometry and goldmann applanation tonometry. J Glaucoma. 2006;15(1):2-6.

29. Elsheikh A, Alhasso D, Rama P. Assessment of the epithelium's contribution to corneal biomechanics. Exp Eye Res. 2008;86(2):445-51.

30. Hjortdal JO. Regional elastic performance of the human cornea. J Biomech. 1996;29(7):931-42

31. Smolek MK. Holographic interferometry of intact and radially incised human eye-bank corneas. J Cataract Refract Surg. 1994;20(3):277-86.

32. Elsheikh A, McMonnies CW, Whitford C, Boneham GC. In vivo study of corneal responses to increased intraocular pressure loading. Eye Vis (Lond). 2015;2:20.

33. Pierscionek BK, Asejczyk-Widlicka M, Schachar RA. The effect of changing intraocular pressure on the corneal and scleral curvatures in the fresh porcine eye. Br J Ophthalmol. 2007;91(6):801-3.

34. McMonnies CW, Boneham GC. Corneal curvature stability with increased intraocular pressure. Eye Contact Lens. 2007;33(3):130-7.

35. Hayes S, Boote C, Lewis J, Sheppard J, Abahussin M, Quantock AJ, et al. Comparative study of fibrillar collagen arrangement in the corneas of primates and other mammals. Vis Biol. 2007;290(12):1542-50.

36. Bell JS, Hayes S, Whitford C, Sanchez-weatherby J, Shebanova O, Vergari C, et al. The hierarchical response of human corneal collagen to load. Acta Biomater. 2018;65:216-25.

37. Gogola A, Jan NJ, Brazile B, Lam P, Lathrop KL, Chan KC, et al. Spatial patterns and age-related changes of the collagen crimp in the human cornea and sclera. Invest Ophthalmol Vis Sci. 2018;59(7):2987-98.

38. Jan NJ, Brazile BL, Hu D, Grube G, Wallace J, Gogola A, et al. Crimp around the globe; patterns of collagen crimp across the corneoscleral shell. Exp Eye Res. 2018;172:159-70.

39. Kamma-Lorger CS, Boote C, Hayes S, Moger J, Burghammer M, Knupp C, et al. Collagen and mature elastic fibre organisation as a function of depth in the human cornea and limbus. J Struct Biol. 2010;169(3):424-30.

40. Lewis PN, White TL, Young RD, Bell JS, Winlove CP, Meek KM. Threedimensional arrangement of elastic fibers in the human corneal stroma. Exp Eye Res. 2016;146:43-53.

41. White $T L$, Lewis PN, Young RD, Kitazawa K, Inatomi T, Kinoshita S, et al. Elastic microfibril distribution in the cornea: differences between normal and keratoconic stroma. Exp Eye Res. 2017;159:40-8.

42. Hogan MJ, Alvarado JA, Wedell JE. Histology of the human eye. Philadelphia: W.B. Saunders; 1971

43. Newton RH, Meek KM. The integration of the corneal and limbal fibrils in the human eye. Biophys J. 1998;75(5):2508-12.

44. Boote $\mathrm{C}$, Hayes S, Abahussin M, Meek KM. Mapping collagen organization in the human cornea: left and right eyes are structurally distinct. Invest Ophthalmol Vis Sci. 2006:47(3):901-8.

45. Kling S, Marcos S. Effect of hydration state and storage media on corneal biomechanical response from in vitro inflation tests. J Refract Surg. 2013; 29(7):490-7.

Ready to submit your research? Choose BMC and benefit from:

- fast, convenient online submission

- thorough peer review by experienced researchers in your field

- rapid publication on acceptance

- support for research data, including large and complex data types

- gold Open Access which fosters wider collaboration and increased citations

- maximum visibility for your research: over $100 \mathrm{M}$ website views per year

At $\mathrm{BMC}$, research is always in progress.

Learn more biomedcentral.com/submissions 